\title{
Very hot tea drinking increases esophageal squamous cell carcinoma risk in a high-risk area of China: a population-based case-control study
}

This article was published in the following Dove Press journal:

Clinical Epidemiology

\author{
Xiaorong Yang, ${ }^{1,2}$ \\ Yingchun $\mathrm{Ni}^{2}$ \\ Ziyu Yuan, ${ }^{3,4}$ \\ Hui Chen,' \\ Amelie Plymoth, ${ }^{5}$ \\ Li Jin, ${ }^{3,4}$ \\ Xingdong Chen, ${ }^{3,4}$ \\ Ming Lu, ${ }^{1,2,4}$ \\ Weimin $\mathrm{Ye}^{4,5}$ \\ 'Clinical Epidemiology Unit, Qilu \\ Hospital of Shandong University, Jinan, \\ China; ${ }^{2}$ Department of Epidemiology, \\ School of Public Health, Shandong \\ University, Jinan, China; ${ }^{3}$ State Key \\ Laboratory of Genetic Engineering, \\ Collaborative Innovation Center for \\ Genetics and Development, School \\ of Life Sciences, Fudan University, \\ Shanghai, China; ${ }^{4}$ Fudan University \\ Taizhou Institute of Health Sciences, \\ Taizhou, China; ${ }^{5}$ Department \\ of Medical Epidemiology and \\ Biostatistics, Karolinska Institutet, \\ Stockholm, Sweden
}

Correspondence: Ming Lu

Clinical Epidemiology Unit, Qilu Hospital of Shandong University, 107 Wenhuaxi

Road, Jinan, Shandong 2500 I2, China

Tel +8653I 82169034

Fax +8653186927544

Email lvming@sdu.edu.cn

Xingdong Chen

State Key Laboratory of Genetic

Engineering, Collaborative Innovation

Center for Genetics and Development,

School of Life Sciences, Fudan University,

Songhu Road 2005, Shanghai 200438,

China

$\mathrm{Tel}+862151630602$

Fax +86 21 51630607

Email xingdongchen@fudan.edu.cn
Background: Previous studies on the association between green tea drinking and esophageal squamous cell carcinoma (ESCC) risk show inconsistent results.

Materials and methods: We conducted a large population-based case-control study from 2010 to 2013 in a high-risk area of China, in which 1,355 ESCC cases and 1,962 controls were recruited. Information on lifelong tea drinking was collected via face-to-face interviews using an electronic structured questionnaire. ORs with $95 \%$ CIs were estimated using unconditional logistic regression models.

Results: Most tea drinkers were males and consumed exclusively green tea. After adjustment for potential confounders, among men the OR of ever green tea drinking for ESCC risk was 1.52 (95\% CI: 1.24-1.85), compared with never tea drinking. The excess risk increased monotonically with earlier age at starting, longer duration, more intensity, and accumulation of tea drinking. The OR of drinking very hot green tea for ESCC risk was 2.15 (95\% CI: 1.52-3.05), compared with never drinking tea. For accumulation of tea drinking and the risk of ESCC, a non-linear relationship was observed. Before the accumulation of tea drinking reached $5 \mathrm{~L} /$ day*years, drinking tea showed a mild protective effect; then the ORs sharply increased to around 2.0 from $5 \mathrm{~L} /$ day*years to $25 \mathrm{~L} /$ day*years, and leveled off thereafter. The non-linear relationship was further modified by tea temperature. The joint effect of tea drinking and alcohol consumption on ESCC risk was also significant $(P=0.019)$.

Conclusion: Very hot tea drinking significantly increases the risk of ESCC among Chinese men, which is particularly evident among alcohol drinkers.

Keywords: green tea, high temperature, esophageal squamous cell carcinoma, risk factor, case-control study

\section{Introduction}

Generally, tea is classified into three main subtypes: non-fermented tea (eg, green tea), semi-fermented tea (eg, oolong tea) and fermented tea (eg, black tea). In 2013, approximately $64 \%$ of tea production worldwide was black tea and $34 \%$ green tea. ${ }^{1}$ Both green tea and black tea, containing plenty of health-related chemical compounds, are considered as health-promoting beverages and believed to be able to prevent the occurrence of cancer, cardiovascular diseases and metabolic abnormalities. ${ }^{2}$ However, Islami et al demonstrated that drinking hot and very hot black tea increased the risk of esophageal squamous cell carcinoma (ESCC) more than 2- and 8-fold, respectively, compared with drinking warm black tea in Iran. ${ }^{3}$ However, the interaction of tea temperature and alcohol drinking was not explored in their study since their study 
participants did not drink alcohol. ${ }^{3}$ A recent report from the Kadoorie cohort revealed a significant alcohol-hot tea interaction on the risk of esophageal cancer. ${ }^{4}$

Although some studies have reported potential chemopreventive properties of tea beverages for cancer, for which black tea is weaker than green tea, ${ }^{5,6}$ the results between green tea and esophageal cancer are controversial in highincidence areas, where ESCC is the overwhelming subtype of esophageal cancer and its precise carcinogenic factors are still unclear. ${ }^{7.8}$ On the other hand, green tea beverage is usually a mixture of tea leaves and boiling water in China, and the potential thermal injury should be considered, because very hot beverages were identified to be Group 2A carcinogens for ESCC by IARC monographs. ${ }^{9}$ Three meta-analyses have been conducted to illustrate the effect of green tea on ESCC development, but the results show severe heterogeneity. ${ }^{10-12}$ Several case-control studies show drinking green tea could marginally decrease the risk of esophageal cancer. ${ }^{13-17}$ On the contrary, the other case-control studies and two cohort studies indicated consuming green tea had a dangerous tendency of increasing esophageal cancer risk. ${ }^{4,18-21}$ The majority of related studies do not take into account the effects of tea beverage temperature, except for two case-control studies and one cohort study, ${ }^{4,20,21}$ Further, most case-control studies recruited esophageal cancer cases weeks or months after diagnosis, thus case subjects might meditate the causes of the disease and exaggerate reporting exposure to some potential risk factors. Further the interaction effects of hot tea drinking and alcohol drinking on ESCC incidence has rarely been reported. ${ }^{4}$

ESCC is the overwhelming subtype of esophageal cancer with more than $95 \%$ proportion in China. ${ }^{22}$ In current analysis, we assessed the association between green tea drinking, tea temperature, and ESCC development, using data collected in a population-based case-control study with a rigorous design to explore the etiology of upper gastrointestinal cancer in Taixing, a high-risk area in China $(54.4 / 100000){ }^{23}$

\section{Materials and methods Study design and participants}

The study was approved by the ethics review boards of School of Life Sciences of Fudan University (date: February 19, 2009), and Qilu Hospital of Shandong University (date: March 8, 2010). All study participants provided written informed consent.

The details of the study design and participant enrollment flow chart have been described previously. ${ }^{24}$ Briefly, we aimed to gather all newly diagnosed esophageal cancer cases in Taixing, China, from October 2010 to September 2013. We limited participants to those aged $40-85$ years, who had lived in Taixing for at least 5 years. When a patient was suspected to have esophageal cancer during endoscopic examination in one of four major local hospitals, we immediately interviewed the patient using an electronic questionnaire (Figure S1). Patients who were not confirmed after histopathological assessment were removed from the study. To identify and interview missed esophageal cancer cases in the four endoscopy units, we double-checked with the local Cancer Registry at the end of each year.

During the 3-year period, we enrolled 1,401 suspected cases from the hospitals' endoscopy units and 280 reported cases via the local Cancer Registry. For each case, we aimed to collect sections from formalin-fixed and paraffinembedded tissue blocks and original pathological reports after surgical resection. After assessing the pathological sections and surgical/pathological reports, 1,499 esophageal cases were confirmed by reviewing pathological sections or surgical/pathological reports. According to the histopathological classification, 1,418 cases of ESCC and 81 cases of non-ESCC were confirmed and enrolled in the study. We estimate that $78.3 \%$ of all incident esophageal cases in the study base were included according to the estimated number from the local Cancer Registry.

During the same period, control participants were randomly selected from Taixing Population Registry, by 5-year age group and sex, for frequency matching to the distribution of the cases. Overall 2,011 control subjects participated (participation rate: $70.4 \%$ ), of whom 19 were out of the eligible age range, so in total 1,992 were included in the current study.

The analysis was based on the 1,418 ESCC cases, which were independently reviewed and confirmed, and 1,992 control subjects. After further excluding 93 subjects with incomplete questionnaire information on tea drinking history, 1,355 cases and 1,962 controls were included in the final analysis.

\section{Exposure assessment}

All participants were interviewed face to face by trained interviewers using an electronic questionnaire, which included demographics, family wealth, family history of cancer, oral hygiene, personal medical history, cigarette smoking history, alcohol and tea drinking history, and dietary history 10 years ago.

\section{Tea drinking history}

This section covers each participant's lifelong history of tea drinking until interview date. Participants were asked about 
the starting age of tea drinking, the age of permanently stopping drinking tea (among ex-drinkers), amount of consumed tea in a typical day (volume), the average number of days per week or per month they drank tea, the usual temperature and concentration of the tea (Supplementary material).

\section{Definition of tea drinking}

In the local area, located in East China Plain, the process of tea brewing is usually to take a certain amount of dry tea leaves into a tea cup/pot, then pour boiling water into it. After cooling tea beverage in room temperature for various minutes, people usually drink it directly. Ever tea drinkers were defined as those who had used the respective tea product for at least 6 months and drank tea at least once per day. Tea ex-drinkers referred to those who had quitted drinking tea at least 2 years before interview date because early uncomfortable symptoms of esophageal cancer might lead patients to stop tea drinking. Duration of tea drinking was defined as the sum of years of drinking tea. Drinking intensity was defined as the average amount (volume) of tea intake per day. We estimated each participant's lifetime cumulative quantity of tea drinking by multiplying the cumulative years with amount of tea drinking per day (L/day), expressed as L/ day*years. According to the time between placing tea leaves mixed with boiling water and tea drinking, the temperature of tea drinking was classified as very hot (less than 1 minute), hot (1-5 minutes) and warm (more than 5 minutes). High concentration was defined as the volume of expanded tea leaves in boiling water more than half of the volume of tea beverage, otherwise it was defined as low concentration (Supplementary material). For data analysis, we categorized the derived continuous variables about tea drinking using approximate quartile cut points, based on the distribution among controls.

\section{Statistical analyses}

Since the prevalence and pattern of tea drinking habits are different between men and women in China, ${ }^{25}$ we analyzed the data for men and women separately. We used unconditional logistic regression models to estimate ORs with $95 \%$ CIs for ESCC in association with tea drinking. Two main models were fitted: the age-adjusted model only adjusted for age (continuous variable), and the fully adjusted model which additionally adjusted for other potential confounders, including marital status, education level, occupation, family wealth score, body mass index 10 years ago, sum of missing and filled teeth, times of tooth brushing per day, smoking pack-years, alcohol consumption intensity, and family history of esophageal cancer among first-degree relatives. We applied multiple correspondence analysis to calculate family wealth score based on ownership of some valuable home appliances, and classified family wealth score into five groups according to quintiles among controls. ${ }^{26}$ Except for tea temperature and concentration, we performed trend tests by using median within each category among all participants and tea drinkers alone, respectively. The non-linear relationship between lifelong accumulation of tea drinking and the risk of ESCC was assessed using a restricted cubic spline regression with five knots. To test the modification effect of the tea-ESCC association by alcohol drinking, we used likelihood ratio test, ie, by comparing nested models and main models. All analyses were carried out using Stata 13.1 (StataCorp LP, College Station, TX, USA). Two-sided $P$-values less than 0.05 were considered statistically significant.

\section{Data availability}

The datasets generated and analyzed during the current study are available from the corresponding authors on reasonable request.

\section{Results}

More than 95\% (923/968) of the tea drinkers of our recruited participants were men, and about 99\% (959/968) of the tea drinkers consumed green tea (Table 1). Thus, the analysis was focused on the effect of green tea beverage on ESCC risk, and the results are presented by sex. To avoid misclassification, nine tea drinkers consuming other tea beverages were excluded in the main analysis.

Table 2 summarizes the characteristics of ESCC cases and controls stratified by sex. Among men, ESCC cases had a lower education level, lower family wealth score, lower body mass index 10 years ago, fewer times of daily tooth brushing, more smoking pack-years, more alcohol consumption, and more frequently reporting a positive family history of esophageal cancer among first-degree relatives, compared to controls. Such differences were similar among women, except for cigarette smoking and alcohol consumption, as most women did not have such habits. In addition, female ESCC cases were a bit older than controls, more likely to be farmers, and had a higher sum of missing and filled teeth. The prevalence of tea drinking habit (with different tea temperature at consumption) by selected risk factors for ESCC in male controls was further analyzed (Table S1). The result showed that except family history of esophageal cancer among first-degree relatives, distribution of tea drinking habit (and tea temperature) was significantly different by other considered factors. 
Table I Tea drinking type distribution of the study participants enrolled in a population-based case-control study on esophageal squamous cell carcinoma in Taixing, China ( $N=3,317)$

\begin{tabular}{|c|c|c|c|c|c|c|}
\hline \multirow[t]{2}{*}{ Variables } & \multicolumn{3}{|c|}{ Men $(n=2,274)$} & \multicolumn{3}{|c|}{ Women $(n=1,043)$} \\
\hline & $\begin{array}{l}\text { Controls } \\
(n=1,353) \\
N(\%)\end{array}$ & $\begin{array}{l}\text { Cases } \\
(n=921) \\
N(\%)\end{array}$ & $P_{\text {-value }}{ }^{a}$ & $\begin{array}{l}\text { Controls } \\
(n=609) \\
N(\%)\end{array}$ & $\begin{array}{l}\text { Cases } \\
(n=434) \\
N(\%)\end{array}$ & $P$-value ${ }^{a}$ \\
\hline \multicolumn{7}{|c|}{ Tea drinking type } \\
\hline Never & $858(63.4 I)$ & $493(53.53)$ & $<0.001$ & $578(94.91)$ & $420(96.77)$ & 0.144 \\
\hline Green tea & $490(36.22)$ & $425(46.15)$ & & $30(4.93)$ & $14(3.23)$ & \\
\hline Oolong tea & $3(0.22)$ & $I(0.1 I)$ & & $0(0.00)$ & $0(0.00)$ & \\
\hline Black tea & $2(0.14)$ & $2(0.22)$ & & $0(0.00)$ & $0(0.00)$ & \\
\hline Flower tea & $0(0.00)$ & $0(0.00)$ & & I (0.16) & $0(0.00)$ & \\
\hline
\end{tabular}

Notes: a $P$-values were derived using Fisher's exact test for each categorical variable, after excluding the corresponding missing value.

Table 2 Demographic and lifestyle information of the study participants enrolled in a population-based case-control study on esophageal squamous cell carcinoma in Taixing, China $(\mathrm{N}=3,308)$

\begin{tabular}{|c|c|c|c|c|c|c|}
\hline \multirow[t]{2}{*}{ Variables } & \multicolumn{3}{|c|}{ Men $(n=2,266)$} & \multicolumn{3}{|c|}{ Women $(n=1,042)$} \\
\hline & $\begin{array}{l}\text { Controls } \\
(n=I, 348) \\
N(\%)\end{array}$ & $\begin{array}{l}\text { Cases } \\
(n=9 \mid 8) \\
N(\%)\end{array}$ & $P$-value ${ }^{a}$ & $\begin{array}{l}\text { Controls } \\
(n=608) \\
N(\%)\end{array}$ & $\begin{array}{l}\text { Cases } \\
(n=434) \\
N(\%)\end{array}$ & $P$-value ${ }^{a}$ \\
\hline Age at interview (mean \pm SD, years) & $65.5 \pm 8.4$ & $65.2 \pm 8.4$ & 0.229 & $67.5 \pm 9.4$ & $69.3 \pm 7.6$ & 0.026 \\
\hline \multicolumn{7}{|l|}{ Age group } \\
\hline $40-49$ & $52(3.86)$ & $30(3.26)$ & 0.238 & $29(4.77)$ & $5(1.15)$ & $<0.001$ \\
\hline $50-59$ & $275(20.40)$ & $202(22.00)$ & & $90(14.80)$ & $35(8.06)$ & \\
\hline $60-69$ & $578(42.88)$ & $406(44.23)$ & & $206(33.88)$ & $177(40.78)$ & \\
\hline 70-79 & $395(29.30)$ & $237(25.82)$ & & $230(37.83)$ & $179(41.24)$ & \\
\hline $80-85$ & $48(3.56)$ & $43(4.68)$ & & $53(8.72)$ & $38(8.76)$ & \\
\hline \multicolumn{7}{|l|}{ Marital status } \\
\hline Unmarried & $63(4.67)$ & $55(5.99)$ & 0.250 & $5(0.82)$ & $2(0.46)$ & 0.516 \\
\hline Married & $\mathrm{I}, \mathrm{I} 4 \mathrm{I}(84.64)$ & $755(82.24)$ & & $422(69.4 I)$ & $291(67.05)$ & \\
\hline Divorced/widowed & $144(10.68)$ & $108(11.76)$ & & I8I (29.77) & I4I (32.49) & \\
\hline \multicolumn{7}{|l|}{ Education level } \\
\hline Illiteracy & $166(12.31)$ & $162(17.65)$ & $<0.001$ & $36 I(59.38)$ & $314(72.35)$ & $<0.001$ \\
\hline Primary school & $574(42.58)$ & $40 I(43.68)$ & & $168(27.63)$ & $103(23.73)$ & \\
\hline Junior high school & $458(33.98)$ & $273(29.74)$ & & $67(11.02)$ & $15(3.46)$ & \\
\hline High school and above & $150(11.13)$ & $82(8.93)$ & & $12(1.97)$ & $2(0.46)$ & \\
\hline \multicolumn{7}{|l|}{ Occupation } \\
\hline Farmer & $695(51.56)$ & $485(52.83)$ & 0.790 & $536(88.16)$ & $410(94.47)$ & 0.002 \\
\hline Worker & $352(26.11)$ & $238(25.93)$ & & $45(7.40)$ & $16(3.69)$ & \\
\hline Service/clerk/professional/administrator & $301(22.33)$ & $195(21.24)$ & & $27(4.44)$ & $8(1.84)$ & \\
\hline \multicolumn{7}{|l|}{ Family wealth score } \\
\hline Q I & $282(20.92)$ & $276(30.07)$ & $<0.001$ & $117(19.24)$ & $119(27.42)$ & 0.001 \\
\hline Q2 & $233(17.28)$ & $162(17.65)$ & & $118(19.41)$ & $88(20.28)$ & \\
\hline Q3 & $284(21.07)$ & $209(22.77)$ & & $144(23.68)$ & $106(24.42)$ & \\
\hline Q4 & $296(21.96)$ & $176(19.17)$ & & $132(2|.7|)$ & $83(19.12)$ & \\
\hline Q5 & $253(18.77)$ & $95(10.35)$ & & $97(15.95)$ & $38(8.76)$ & \\
\hline \multicolumn{7}{|l|}{ Body mass index 10 years ago $\left(\mathrm{kg} / \mathrm{m}^{2}\right)$} \\
\hline$<18.5$ (Lean) & $57(4.23)$ & $68(7.4 I)$ & $<0.001$ & $52(8.55)$ & $57(13.13)$ & 0.002 \\
\hline$[18.5,24)$ (Normal) & $848(62.91)$ & $622(67.76)$ & & $333(54.77)$ & $242(55.76)$ & \\
\hline$[24,28)$ (Overweight) & $360(26.7 I)$ & $189(20.59)$ & & $177(29.11)$ & $122(28.11)$ & \\
\hline$\geq 28$ (Obesity) & $82(6.08)$ & $39(4.25)$ & & $46(7.57)$ & $13(3.00)$ & \\
\hline Missing & $\mathrm{I}(0.07)$ & $0(0.00)$ & & & & \\
\hline
\end{tabular}


Table 2 (Continued)

\begin{tabular}{|c|c|c|c|c|c|c|}
\hline \multirow[t]{2}{*}{ Variables } & \multicolumn{3}{|c|}{ Men $(n=2,266)$} & \multicolumn{3}{|c|}{ Women $(n=1,042)$} \\
\hline & $\begin{array}{l}\text { Controls } \\
(n=1,348) \\
N(\%)\end{array}$ & $\begin{array}{l}\text { Cases } \\
(n=9 \mid 8) \\
N(\%)\end{array}$ & $P$-value ${ }^{a}$ & $\begin{array}{l}\text { Controls } \\
(n=608) \\
N(\%)\end{array}$ & $\begin{array}{l}\text { Cases } \\
(n=434) \\
N(\%)\end{array}$ & P-value ${ }^{a}$ \\
\hline \multicolumn{7}{|c|}{ Sum of missing and filled teeth } \\
\hline None & $370(27.45)$ & $240(26.14)$ & 0.212 & $130(2 \mid .38)$ & $57(13.13)$ & $<0.001$ \\
\hline$<6$ & $509(37.76)$ & $325(35.40)$ & & $201(33.06)$ & $13 \mid(30.18)$ & \\
\hline$\geq 6$ & $463(34.35)$ & $348(37.91)$ & & $276(45.39)$ & $237(54.6 \mathrm{I})$ & \\
\hline Missing & $6(0.45)$ & $5(0.54)$ & & I (0.16) & $9(2.07)$ & \\
\hline \multicolumn{7}{|c|}{ Number of tooth brushing per day } \\
\hline$<2$ & $892(66.17)$ & $743(80.94)$ & $<0.001$ & $386(63.49)$ & $348(80.18)$ & $<0.001$ \\
\hline$\geq 2$ & $450(33.38)$ & $172(18.74)$ & & $222(36.5 I)$ & 85 (19.59) & \\
\hline Missing & $6(0.45)$ & $3(0.33)$ & & $0(0.00)$ & $\mathrm{I}(0.23)$ & \\
\hline \multicolumn{7}{|c|}{ Smoking (pack-years) } \\
\hline Never & $298(22.11)$ & $153(16.67)$ & $<0.001$ & $585(96.22)$ & $413(95.16)$ & 0.846 \\
\hline$\leq 30$ & $521(38.65)$ & $324(35.29)$ & & $19(3.13)$ & $15(3.46)$ & \\
\hline$>30$ & $526(39.02)$ & $436(47.49)$ & & $4(0.66)$ & $4(0.92)$ & \\
\hline Missing & $3(0.22)$ & $5(0.54)$ & & $0(0.00)$ & $2(0.46)$ & \\
\hline \multicolumn{7}{|c|}{ Alcohol drinking intensity (g/day) } \\
\hline Never & $582(43.18)$ & $235(25.60)$ & $<0.001$ & $573(94.24)$ & $400(92.17)$ & 0.308 \\
\hline$\leq 80$ & $372(27.60)$ & $288(31.37)$ & & $31(5.10)$ & $31(7.14)$ & \\
\hline$>80$ & $393(29.15)$ & $395(43.03)$ & & $3(0.49)$ & I $(0.23)$ & \\
\hline Missing & I (0.07) & $0(0.00)$ & & $I(0.16)$ & $2(0.46)$ & \\
\hline \multicolumn{7}{|c|}{$\begin{array}{l}\text { Family history of esophageal cancer } \\
\text { among first-degree relatives }\end{array}$} \\
\hline No & $\mathrm{I}, 096(8 \mathrm{I} .3 \mathrm{I})$ & $621(67.65)$ & $<0.001$ & $495(8 I .4 I)$ & $294(67.74)$ & $<0.00$ I \\
\hline Yes & $249(18.47)$ & $296(32.24)$ & & $112(18.42)$ & $139(32.03)$ & \\
\hline Missing & $3(0.22)$ & I (0.II) & & I (0.16) & $\mathrm{I}(0.23)$ & \\
\hline
\end{tabular}

Notes: ${ }^{2}$-values were derived using chi-squared test for each categorical variable and Wilcoxon rank sum test for continuous variable, after excluding the corresponding missing value.

Table 3 shows the association of green tea drinking with the risk of ESCC among men. The age-adjusted and fully adjusted results indicate that ever green tea drinking increased 1.5-fold risk of ESCC, compared with never tea drinking, and the population attributable fraction of tea drinking for the risk of ESCC in men was $15.90 \%$. The excess ESCC risk in association with green tea drinking was more evident among those who started drinking tea at an earlier age, had a longer duration of green tea drinking, drank more green tea per day, or had a higher cumulative dose (L/day*years) of green tea drinking. Similarly, the excess risks increased monotonically with increasing tea temperature at consumption (fully-adjusted model, OR=1.29, 1.47 and 2.15 for warm, hot and very hot tea temperature, respectively; $P$-value for trend $<0.001$ ). However, ORs did not differ substantially for different tea concentrations.

The proportion of ever green tea drinking among females was $4.22 \%$ (44/1042), and the majority of those tea drinkers started drinking tea after 50 years of age (27/44), had a duration of tea drinking no more than 12 years $(24 / 44)$, consumed no more than $500 \mathrm{~mL}$ per day $(25 / 44)$, had no more than $8 \mathrm{~L} /$ day*years (25/44) of cumulative exposure, and drank tea with warm temperature (29/44) or low concentration (35/44). The fully adjusted OR for ESCC in relation to tea drinking among females was 0.79 (95\% CI: 0.39-1.61).

Figure 1A shows the non-linear relationship between accumulation of tea drinking and the risk of ESCC among men. Before the accumulation of tea drinking reached 10 L/day*years, drinking tea showed a U-shape relation with ESCC risk, with the nadir at $5 \mathrm{~L} /$ day*years; after that the ORs sharply rose to around 2.0 at $25 \mathrm{~L} /$ day*years, then leveled off. Figure 1B further shows the non-linear relationship between accumulation of tea drinking and the risk of ESCC, stratified by tea temperature. The previously observed U-shaped relation was evident only among those drinking tea at warm temperature. However, for both drinking warm and hot tea, the relative risk increased to around 2 , and leveled off at about $25 \mathrm{~L} /$ day*years. The excess risk was most evident for drinking 
Table 3 ORs and $95 \% \mathrm{Cls}$ for esophageal squamous cell carcinoma in association with green tea drinking among men by unconditional logistic regression $(\mathrm{N}=2,266)^{\mathrm{a}}$

\begin{tabular}{|c|c|c|c|c|}
\hline Variables & $\begin{array}{l}\text { Controls } \\
\text { N (\%) }\end{array}$ & $\begin{array}{l}\text { Cases } \\
\mathbf{N}(\%)\end{array}$ & $\begin{array}{l}\text { Age-adjusted } \\
\text { OR (95\% Cls) }\end{array}$ & $\begin{array}{l}\text { Fully adjusted } \\
\text { OR }(95 \% \mathrm{Cls})\end{array}$ \\
\hline \multicolumn{5}{|l|}{ Green tea drinking } \\
\hline Never & $858(63.65)$ & $493(53.70)$ & 1.00 (reference) & $\mathrm{I} .00$ (reference) \\
\hline Ever & $490(36.35)$ & $425(46.30)$ & $1.51(1.27-1.79)$ & $1.52(1.24-1.85)$ \\
\hline \multicolumn{5}{|c|}{ Age at starting drinking tea (years) } \\
\hline$\geq 50$ & $136(10.09)$ & $99(10.78)$ & $1.26(0.95-1.68)$ & $1.19(0.87-1.62)$ \\
\hline$(36,50)$ & $116(8.61)$ & $94(10.24)$ & $1.42(1.05-1.91)$ & $1.35(0.98-1.87)$ \\
\hline$(27,36)$ & $117(8.68)$ & $110(11.98)$ & $1.64(1.24-2.19)$ & $1.93(1.39-2.67)$ \\
\hline$<27$ & $121(8.98)$ & $122(13.29)$ & $1.77(1.34-2.33)$ & $1.85(1.35-2.53)$ \\
\hline$P$ for trend among all subjects & & & $<0.001$ & $<0.001$ \\
\hline$P$ for trend among tea drinkers & & & 0.063 & 0.037 \\
\hline \multicolumn{5}{|c|}{ Duration of drinking tea (years) } \\
\hline$\leq 12$ & $122(9.05)$ & $76(8.28)$ & $1.08(0.80-1.47)$ & $0.98(0.70-1.37)$ \\
\hline$(12,25)$ & $128(9.50)$ & $122(13.29)$ & $1.66(1.26-2.18)$ & $1.68(1.25-2.28)$ \\
\hline$(25,35)$ & $124(9.20)$ & $118(12.85)$ & $1.65(1.25-2.18)$ & $1.76(1.29-2.4 \mathrm{I})$ \\
\hline$\geq 35$ & $116(8.61)$ & $109(11.87)$ & $1.64(1.23-2.18)$ & $1.79(1.29-2.48)$ \\
\hline$P$ for trend among all subjects & & & $<0.001$ & $<0.001$ \\
\hline$P$ for trend among tea drinkers & & & 0.040 & 0.013 \\
\hline \multicolumn{5}{|c|}{ Intensity of drinking tea (mL/day) } \\
\hline$\leq 500$ & $138(10.24)$ & $80(8.7 I)$ & $1.01(0.75-1.36)$ & $0.98(0.7|-| .36)$ \\
\hline$(500,800)$ & III (8.23) & $107(11.66)$ & $1.68(1.26-2.24)$ & $1.78(1.29-2.44)$ \\
\hline$(800,1,400)$ & $97(7.20)$ & $102(11.11)$ & $1.83(1.36-2.48)$ & $1.72(1.23-2.40)$ \\
\hline$>1,400$ & $144(10.68)$ & $136(14.81)$ & $1.65(1.27-2.14)$ & $1.75(1.3 \mathrm{I}-2.35)$ \\
\hline$P$ for trend among all subjects & & & $<0.001$ & $<0.001$ \\
\hline$P$ for trend among tea drinkers & & & 0.026 & 0.024 \\
\hline \multicolumn{5}{|c|}{ Accumulation of tea drinking (L/day*year) } \\
\hline$\leq 8$ & $121(8.98)$ & $80(8.7 I)$ & $1.15(0.85-1.56)$ & $\mathrm{I} .08(0.78-\mathrm{I} .5 \mathrm{I})$ \\
\hline$(8,18)$ & $122(9.05)$ & $89(9.69)$ & $1.27(0.94-1.7 \mathrm{I})$ & $1.25(0.90-1.73)$ \\
\hline$(18,36)$ & $124(9.20)$ & $131(14.27)$ & $1.84(1.40-2.4 I)$ & $1.94(1.43-2.62)$ \\
\hline$>36$ & $123(9.12)$ & $125(13.62)$ & $1.77(1.35-2.32)$ & $1.93(1.4 \mathrm{I}-2.64)$ \\
\hline$P$ for trend among all subjects & & & $<0.001$ & $<0.001$ \\
\hline$P$ for trend among tea drinkers & & & 0.014 & 0.006 \\
\hline \multicolumn{5}{|l|}{ Tea temperature } \\
\hline Warm & $205(15.21)$ & $148(16.12)$ & $1.25(0.99-1.59)$ & $1.29(0.99-1.69)$ \\
\hline Hot & $210(15.58)$ & $175(19.06)$ & $1.45(1.15-1.82)$ & $1.47(1.14-1.91)$ \\
\hline Very hot & $75(5.56)$ & $102(11.11)$ & $2.36(1.72-3.25)$ & $2.15(1.52-3.05)$ \\
\hline$P$ for trend among all subjects & & & $<0.001$ & $<0.001$ \\
\hline$P$ for trend among tea drinkers & & & 0.001 & 0.007 \\
\hline \multicolumn{5}{|l|}{ Tea concentration } \\
\hline Low & $263(19.52)$ & $216(23.53)$ & $1.43(1.16-1.77)$ & $1.54(1.22-1.96)$ \\
\hline High & $226(16.78)$ & $209(22.77)$ & $1.61(1.29-2.00)$ & $1.49(1.16-1.91)$ \\
\hline Missing & $\mathrm{I}(0.07)$ & & & \\
\hline$P$ for trend among all subjects & & & $<0.001$ & $<0.001$ \\
\hline$P$ for trend among tea drinkers & & & 0.381 & 0.708 \\
\hline
\end{tabular}

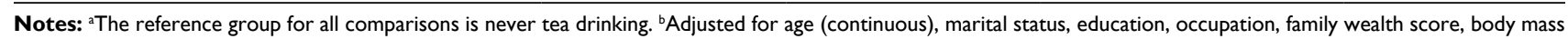
index 10 years ago, sum of missing and filled teeth, number of tooth brushing per day, smoking pack-years, alcohol consumption intensity and family history of esophageal cancer among first-degree relatives (except age, other variables are categorized as shown in Table 2).

very hot tea, with the peak $(\mathrm{OR}>3)$ reached quickly at $20 \mathrm{~L} /$ day*years. Although the relative risks decreased somewhat later, they increased steadily after $50 \mathrm{~L} /$ day*years.

Table 4 presents joint effects of tea drinking and alcohol drinking on ESCC occurrence among men. Subjects who had both tea and alcohol drinking habits had a more than 3-fold relative risk compared with those who abstained from tea and alcohol drinking (OR=3.15, 95\% CI: 2.43-4.09, $P$-value of interaction=0.019). Further we analyzed the interaction between tea drinking temperature and alcohol drinking intensity. Although the interaction test was not statistically significant due to limited sample size ( $P$-value of 

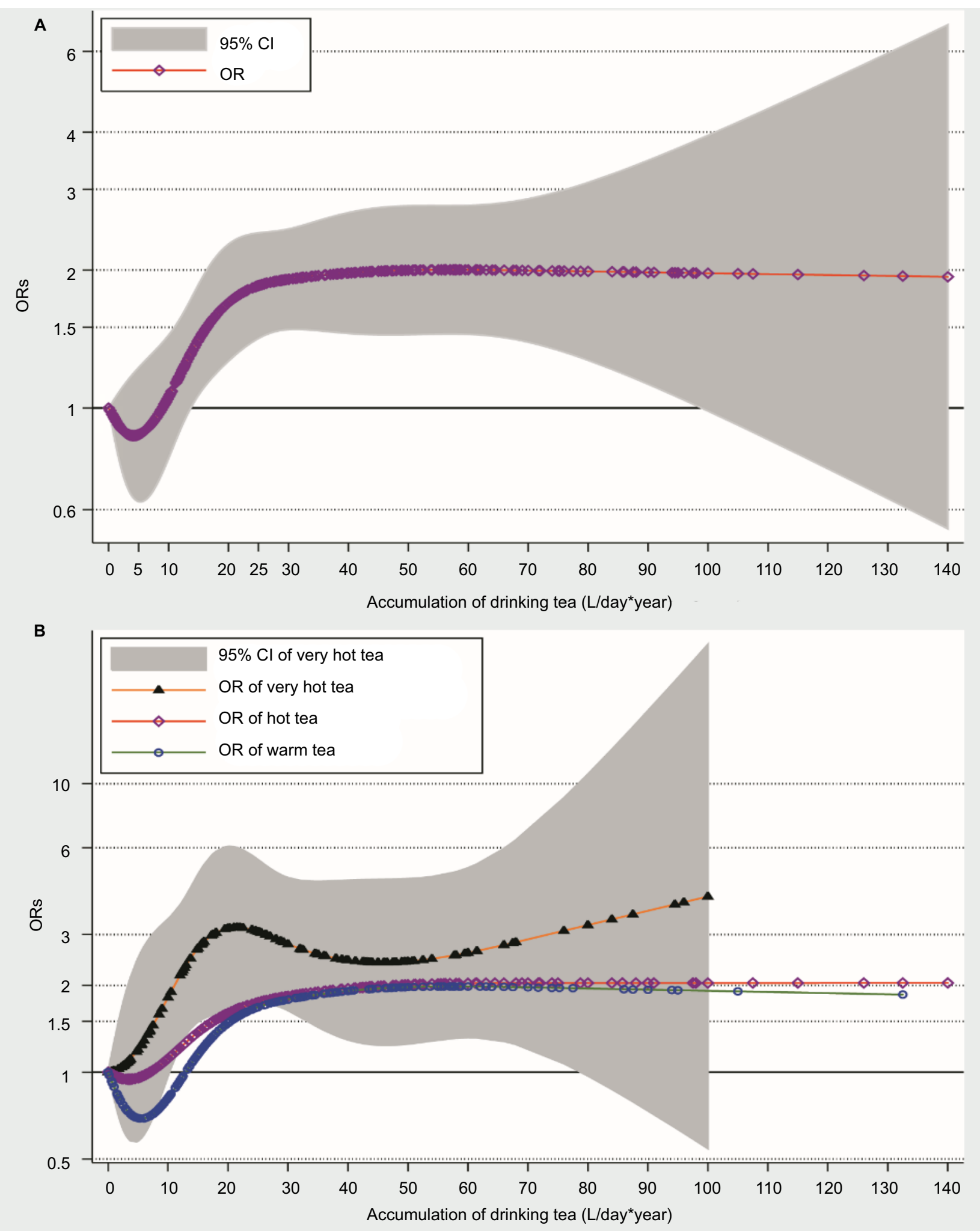

Figure I (A) The association between accumulation of tea drinking and the risk of ESCC among men by restricted cubic spline regression. (B) The association between accumulation of tea drinking and the risk of ESCC among men by tea temperature by restricted cubic spline regression. 
interaction $=0.183$ ), there was a tendency of increasing excess risks with higher temperature of tea drinking and intensity of alcohol drinking jointly $(\mathrm{OR}=6.09,95 \% \mathrm{CI}$ : $3.55-10.43$, for those drinking very hot tea and consuming alcohol more than $80 \mathrm{~g} /$ day, compared with abstainers from both tea and alcohol, Table 5). We also examined the interaction between green tea drinking and some other selected risk factors on the risk of ESCC (Figure S2). Except a borderline significant interaction between tooth brushing times per day, there was no significant interactions between green tea drinking and sum of missing and filled teeth, smoking, and family history of esophageal cancer among first-degree relatives.

A sensitivity analysis was performed by excluding 124 male cases and 33 female cases, enrolled from the local Cancer Registry. The results did not change significantly. For example, compared with never tea drinking, the fully adjusted OR for ESCC in relation to tea drinking was 1.59 (95\% CI: 1.29-1.96) among men. In addition, since there were eight men and one woman who consumed other tea beverages, we did another sensitivity analysis by analyzing all tea drinkers. The results did not change substantially, however.

\section{Discussion}

In this large population-based case-control study conducted in a high-incidence area of China, we observed adverse effects of green tea consumption on the risk of ESCC among men, which is mainly due to very hot temperature of green tea beverage. In general, a monotonic increasing risk of ESCC was observed with earlier age at start, longer duration, larger volume, and higher temperature of green tea drinking, except that consuming warm tea with limited volume marginally prevented ESCC development. In addition, alcohol drinking further increased the harmful effect of very hot green tea drinking on ESCC occurrence.

Only two previous studies about green tea drinking and the risk of esophageal cancer have considered tea temperature, and both of them reported that drinking tea at high temperatures significantly increases esophageal cancer incidence. ${ }^{20,21}$ This is consistent with our main results. Further we found that the tea temperature might play a relatively important role in the relationship between tea drinking and the risk of ESCC, especially when the lifelong accumulation of tea drinking was less than $10 \mathrm{~L} /$ day*years: drinking hot

Table 4 The joint effects of tea drinking and alcohol drinking on esophageal squamous cell carcinoma risk among men by likelihood ratio test $(\mathrm{N}=2,266)$

\begin{tabular}{|c|c|c|c|c|c|}
\hline Tea drinking & $\begin{array}{l}\text { Alcohol } \\
\text { drinking }\end{array}$ & $\begin{array}{l}\text { Controls } \\
\text { N (\%) }\end{array}$ & $\begin{array}{l}\text { Cases } \\
\mathbf{N}(\%)\end{array}$ & $\begin{array}{l}\text { Age-adjusted OR } \\
\text { (95\% Cls) }\end{array}$ & $\begin{array}{l}\text { Fully adjusted OR } \\
(95 \% \mathrm{Cls})^{\mathrm{a}}\end{array}$ \\
\hline & Missing & I (0.07) & $0(0.00)$ & & \\
\hline Never & Never & $423(31.38)$ & 178 (19.39) & I.00 (reference) & I.00 (reference) \\
\hline Ever & Never & $159(11.80)$ & $57(6.21)$ & $0.86(0.60-1.22)$ & $1.06(0.73-1.54)$ \\
\hline Never & Ever & $434(32.20)$ & $315(34.31)$ & $1.73(1.38-2.17)$ & $1.80(1.4 \mid-2.30)$ \\
\hline Ever & Ever & 331 (24.55) & $368(40.09)$ & $2.66(2.11-3.36)$ & $3.15(2.43-4.09)$ \\
\hline$P$ of interaction & & & & 0.004 & 0.019 \\
\hline
\end{tabular}

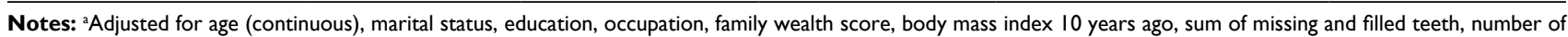
tooth brushing per day, smoking pack-years, and family history of esophageal cancer among first-degree relatives (except age, other variables are categorized as shown in Table 2).

Table 5 The joint effects of tea drinking temperature and alcohol drinking intensity on esophageal squamous cell carcinoma risk among men by likelihood ratio test $(\mathrm{N}=2,266)^{\mathrm{a}}$

\begin{tabular}{|c|c|c|c|c|c|c|c|}
\hline \multirow{3}{*}{$\begin{array}{l}\text { Tea } \\
\text { temperature }\end{array}$} & \multicolumn{6}{|c|}{ Alcohol drinking intensity (g/day) } & \multirow[t]{3}{*}{$P$ of trend } \\
\hline & \multicolumn{2}{|l|}{ Never } & \multicolumn{2}{|l|}{$\leq 80$} & \multicolumn{2}{|l|}{$>80$} & \\
\hline & $\begin{array}{l}\text { Control } \\
\text { s/cases }\end{array}$ & OR (95\% Cls) & $\begin{array}{l}\text { Control } \\
\text { s/cases }\end{array}$ & OR (95\% Cls) & $\begin{array}{l}\text { Control } \\
\text { s/cases }\end{array}$ & OR (95\% Cls) & \\
\hline Never & $423 / 178$ & 1.00 (reference) & $232 / 159$ & $1.74(1.31-2.31)$ & $202 / 156$ & $1.86(1.39-2.50)$ & $<0.001$ \\
\hline Warm & $59 / 17$ & $0.90(0.49-1.63)$ & $69 / 43$ & $2.08(1.32-3.28)$ & $77 / 88$ & $3.11(2.12-4.58)$ & $<0.001$ \\
\hline Hot & $69 / 26$ & $1.08(0.65-1.81)$ & $51 / 60$ & $3.09(1.98-4.81)$ & $90 / 89$ & $3.02(2.08-4.40)$ & 0.002 \\
\hline Very hot & $31 / 14$ & $1.26(0.63-2.52)$ & $20 / 26$ & $3.43(1.80-6.55)$ & $24 / 62$ & $6.09(3.55-10.43)$ & $<0.001$ \\
\hline$P$ of trend & & 0.410 & & 0.014 & & $<0.001$ & \\
\hline$P$ of interaction & 0.183 & & & & & & \\
\hline
\end{tabular}

Notes: ${ }^{a}$ Adjusted for age (continuous), marital status, education, occupation, family wealth score, body mass index 10 years ago, sum of missing and filled teeth, number of tooth brushing per day, smoking pack-years, and family history of esophageal cancer among first-degree relatives (except age, other variables are categorized as shown in Table 2). 
or very hot tea beverage showed a harmful effect on ESCC development; however, consuming warm tea marginally prevented ESCC development.

Previous studies did not observe a significant interaction between tea drinking and alcohol consumption on the risk of ESCC, but they all pointed out a potential joint effect: co-exposure usually has the highest excess risk. ${ }^{19-21}$ In our study, we found a significant interaction between ever tea drinking and ever alcohol consumption on ESCC risk. Although further exploring the interaction between tea temperature and alcohol drinking intensity resulted in statistical non-significance due to a limited sample size, the trend was evident especially among heavy alcohol consumers. To corroborate this, recently a cohort study conducted in China reported a significant interaction between hot tea consumption and alcohol use on the risk of esophageal cancer. ${ }^{4}$ When most Chinese men drink strong Chinese spirits, they usually consume relatively hot tea beverage to relieve the stimulation of ethanol. The thermal injury may damage the esophageal mucosa and its barrier function, which subsequently intensifies the harmful effects from other risk factors. ${ }^{27}$ Thus, the co-exposure of alcohol and hot tea beverage at the same time could lead to an evident harmful effect on the esophageal mucosa.

Experimental and epidemiological studies have reported that green tea with its antimicrobial, anti-oxidative and antiinflammatory activities may be considered as a potential cancer preventive agent. ${ }^{28,29}$ The mild anticancer effect of green tea for the esophagus could be observed only among participants consuming tea beverage with warm temperature in our study. More and more studies demonstrate that consuming hot beverages and food is associated with an increased risk of esophageal cancer, particularly ESCC. ${ }^{27,30,31}$ The N-nitroso compounds released during inflammatory processes as a result of thermal injury may contribute to the carcinogenesis of the esophagus..$^{32}$ In Chinese tea drinking culture, most tea drinkers usually consume tea beverage with a relative high temperature, ${ }^{20,21}$ and even warm tea may have a temperature higher than the tolerance of esophageal epithelial cells. In such situations, the hot green tea may be a proxy for drinking hot water, and the potential anticancer effect of green tea may be masked by the damage of hot beverage on esophageal epithelium. Therefore, the observed association between green tea drinking and the risk of ESCC may result from the high temperature rather than the green tea. The appropriate tea temperature for healthy lifestyle needs be elucidated in future.
In our study, the proportion of female tea drinker was too low, and the estimated OR for women might not be reliable. The possible reason of female participants rarely drinking tea is that the study area does not produce tea. Thus, tea drinking behavior, like smoking and alcohol drinking, usually stands for a special social currency there. For the birth cohorts the study subjects belong, men usually played a leading role in social interactions due to Chinese traditional culture. Therefore, few women would develop a tea drinking habit spontaneously in the local area. ${ }^{33}$

Our study has several advantages. To decrease recall bias, most cases were interviewed before having been diagnosed with the disease. Other advantages include the relatively large sample size, the independent diagnosis confirmation for all cases, the relatively high response rates for both cases and controls, and the systematic collection of lifelong and detailed tea consumption data. In addition, we randomly selected control participants from the total registry of residents of the study area.

Despite these advantages, several disadvantages should be considered. First, despite our best efforts to contact all eligible study participants, around $30 \%$ of eligible participants were not engaged in our research. Most missing cases were diagnosed in some large-scale hospitals in other big cities, thus the missing cases might have a better family wealth. The missing controls were due to death prior to contact, outmigration, inability to be reached or unwillingness to participate. Therefore, the potential selection bias might be an issue. However, respondents and non-respondents did not show significant differences in regard to age and sex distribution. Second, the temperature of tea beverage was not measured by instruments, and the possible misclassification of tea temperature is an important issue. In general, the tea brewing methods and tea drinking culture in the local area, a small city, were relatively unified and stable, so the cooling time was an indicator of tea temperature. Previous studies have reported that the temperature of tea beverage by the questionnaire using cooling time has a good consistence with the measured centigrade in local areas, ${ }^{3,21}$ which partially eases our concerns of the misclassification of temperature grade. Third, other related information, such as the temperature of daily food and other beverages, like rice porridge, sour porridge, flour soup, noodles and wonton soup, etc., which might modify tea drinking's effect on ESCC risk, was not collected. Moreover, although we interviewed most cases as early as possible to decrease the influence of recall bias, recall bias cannot be completely eliminated in all case-control studies. Therefore, we 
conducted a sensitivity analysis by excluding cases enrolled via the local Cancer Registry, who were interviewed after diagnosis or even treatment. The sensitivity analysis showed similar results as the main analysis including all case subjects, which allayed our concerns of recall bias to a certain extent. However, individuals who underwent endoscopy generally had an indication warranting ESCC, thus recall bias is likely to remain an issue. Finally, this study was conducted in an ESCC high-incidence area in China, and the generalizability of the study results might be limited. But our results indicated that exposure to the high temperature of beverage might be a risk factor, especially in high-incidence areas.

\section{Conclusion}

In summary, very hot green tea drinking significantly increases the risk of ESCC among Chinese men, which is strengthened by consuming alcohol. Our results emphasize that removing or minimizing exposures to these easily modifiable lifestyle risk factors should be added into primary prevention strategies for reducing the ESCC incidence, especially in high-risk areas.

\section{Acknowledgments}

The authors would like to acknowledge the interviewers and technicians at Fudan University Taizhou Institute of Health Sciences for their invaluable contribution to data collection and sample preparation, the staff at the Taixing Center for Disease Control and Prevention for help with organizing the field work, and the staff at Taixing People's Hospital for their assistance with sample collection. Grants were received from the National Natural Science Foundation of China (81573229, 81502870 and 81273151), International S\&T Cooperation Program of China (2015DFE32790), key basic research grants from Science and Technology Commission of Shanghai Municipality (16JC1400500), and National Key Research and Development program of China (2017YFC0907002 and 2017YFC0907501).

\section{Disclosure}

The authors report no conflicts of interest in this work.

\section{References}

1. Chang K. World tea production and trade: current and future development. Rome, Italy: Food and Agriculture Organization of the United Nations; 2015.

2. Khan N, Mukhtar H. Tea and health: studies in humans. Curr Pharm Des. 2013;19(34):6141-6147.

3. Islami F, Pourshams A, Nasrollahzadeh D, et al. Tea drinking habits and oesophageal cancer in a high risk area in northern Iran: population based case-control study. BMJ. 2009;338:b929.
4. Yu C, Tang H, Guo Y, et al. Hot tea consumption and its interactions with alcohol and tobacco use on the risk for esophageal cancer: a population-based cohort study. Ann Intern Med. 2018;168(7):489.

5. Zhou Q, Li H, Zhou JG, Ma Y, Wu T, Ma H. Green tea, black tea consumption and risk of endometrial cancer: a systematic review and meta-analysis. Arch Gynecol Obstet. 2016;293(1):143-155.

6. Henning SM, Wang P, Said JW, et al. Randomized clinical trial of brewed green and black tea in men with prostate cancer prior to prostatectomy. Prostate. 2015;75(5):550-559.

7. Kamangar F, Chow WH, Abnet CC, Dawsey SM. Environmental causes of esophageal cancer. Gastroenterol Clin North Am. 2009;38(1):27-57.

8. Stewart B, Wild CP. World Cancer Report 2014: World Health Organization; 2016.

9. IARC. List of Classifications by cancer sites with sufficient or limited evidence in humans, Volumes $1-121 ; 2018$. Available from: http:// monographs.iarc.fr/ENG/Classification/Table4.pdf.

10. Zheng JS, Yang J, Fu YQ, Huang T, Huang YJ, Li D. Effects of green tea, black tea, and coffee consumption on the risk of esophageal cancer: a systematic review and meta-analysis of observational studies. Nutr Cancer. 2013;65(1):1-16.

11. Sang LX, Chang B, Li XH, Jiang M. Green tea consumption and risk of esophageal cancer: a meta-analysis of published epidemiological studies. Nutr Cancer. 2013;65(6):802-812.

12. Zheng P, Zheng HM, Deng XM, Zhang YD. Green tea consumption and risk of esophageal cancer: a meta-analysis of epidemiologic studies. BMC Gastroenterol. 2012;12:165.

13. Gao YT, Mclaughlin JK, Blot WJ, Ji BT, Dai Q, Fraumeni JF. Reduced risk of esophageal cancer associated with green tea consumption. $\mathrm{J} \mathrm{Natl}$ Cancer Inst. 1994;86(11):855-858.

14. Takezaki T, Shinoda M, Hatooka S, et al. Subsite-specific risk factors for hypopharyngeal and esophageal cancer (Japan). Cancer Causes Control. 2000;11(7):597-608.

15. Gao CM, Takezaki T, Wu JZ, et al. Glutathione-S-transferases M1 (GSTM1) and GSTT1 genotype, smoking, consumption of alcohol and tea and risk of esophageal and stomach cancers: a case-control study of a high-incidence area in Jiangsu Province, China. Cancer Lett. 2002;188(1-2):95-102.

16. Wang Z, Tang L, Sun G, et al. Etiological study of esophageal squamous cell carcinoma in an endemic region: a population-based case control study in Huaian, China. BMC Cancer. 2006;6:287.

17. Chen YK, Lee $\mathrm{CH}, \mathrm{Wu}$ IC, et al. Food intake and the occurrence of squamous cell carcinoma in different sections of the esophagus in Taiwanese men. Nutrition. 2009;25(7-8):753-761.

18. Wang JM, Xu B, Rao JY, Shen HB, Xue HC, Jiang QW. Diet habits, alcohol drinking, tobacco smoking, green tea drinking, and the risk of esophageal squamous cell carcinoma in the Chinese population. Eur J Gastroenterol Hepatol. 2007;19(2):171-176.

19. Ishikawa A, Kuriyama S, Tsubono Y, et al. Smoking, alcohol drinking, green tea consumption and the risk of esophageal cancer in Japanese men. J Epidemiol. 2006;16(5):185-192.

20. Wu M, Liu AM, Kampman E, et al. Green tea drinking, high tea temperature and esophageal cancer in high- and low-risk areas of Jiangsu Province, China: a population-based case-control study. Int $J$ Cancer. 2009;124(8):1907-1913.

21. ChenZ, Chen Q, Xia H,Lin J. Green tea drinking habits and esophageal cancer in southern China: a case-control study. Asian Pac J Cancer Prev. 2011; 12(1):229-233.

22. Arnold M, Soerjomataram I, Ferlay J, Forman D. Global incidence of oesophageal cancer by histological subtype in 2012. Gut. 2015;64(3):381-387.

23. Chen W, HeY, Zheng R, et al. Esophageal cancer incidence and mortality in China, 2009. J Thorac Dis. 2013;5(1):19-26.

24. Yang X, Chen X, Zhuang M, et al. Smoking and alcohol drinking in relation to the risk of esophageal squamous cell carcinoma: a populationbased case-control study in China. Sci Rep. 2017;7(1):17249. 
25. Li X, Lyu J, Guo Y, et al. Regional differences in adults' tea drinking pattern: findings from China Kadoorie Biobank study in 10 areas in China. Chin J Epidemiol. 2015;36(11):1200-1204.

26. Chen X, Yuan Z, Lu M, Zhang Y, Jin L, Ye W. Poor oral health is associated with an increased risk of esophageal squamous cell carcinoma - a population-based case-control study in China. Int J Cancer. 2017;140(3):626-635.

27. Andrici J, Eslick GD. Hot food and beverage consumption and the risk of esophageal cancer: a meta-analysis. Am J Prev Med. 2015;49(6):952-960.

28. Vuong QV. Epidemiological evidence linking tea consumption to human health: a review. Crit Rev Food Sci Nutr. 2014;54(4):523-536.

29. Suzuki Y, Miyoshi N, Isemura M. Health-promoting effects of green tea. Proceedings of the Japan Academy Series B. Phys Biol Sci. 2012;88(3):88-101.
30. Lin J, Zeng R, Cao W, Luo R, Chen J, Lin Y. Hot beverage and food intake and esophageal cancer in southern China. Asian Pac J Cancer Prev. 2011;12(9):2189-2192.

31. Chen Y, Tong Y, Yang C, et al. Consumption of hot beverages and foods and the risk of esophageal cancer: a meta-analysis of observational studies. BMC Cancer. 2015;15:449.

32. Mirvish SS. Role of N-nitroso compounds (NOC) and N-nitrosation in etiology of gastric, esophageal, nasopharyngeal and bladder cancer and contribution to cancer of known exposures to NOC. Cancer Lett. 1995;93(1):17-48.

33. Guan X, Yang J, Xie X, Lin L. Research on the status of Chinese tea consumption and factors of tea consumers' behavior (in Chinese). JTea Sci. 2011;31(6):546-551. 


\section{Supplementary material}

\section{Questionnaire of tea drinking and the related sample images}

All participants were interviewed face to face by trained interviewers using an electronic questionnaire. The translated questionnaire of tea drinking and the corresponding sample images are listed in Figure S1 below.

$\mathbf{A}$

\section{Part VI History of tea drinking}

\section{History of drinking tea}

6.2 Do you have a habit of drinking tea? (At least one cup per day for 6 months) (1) Yes, (2) No $\left.\right|_{-} \mid$

\begin{tabular}{|c|c|c|c|c|c|c|}
\hline & $\begin{array}{l}\text { Size of tea cup } \\
\text { (1) small cup }(50 \mathrm{~mL}) \\
\text { (2) medium cup }(100 \mathrm{~mL}) \\
\text { (3) large cup }(250 \mathrm{~mL})\end{array}$ & $\begin{array}{l}\text { Age at } \\
\text { start }\end{array}$ & $\begin{array}{l}\text { Age at } \\
\text { quitting }\end{array}$ & $\begin{array}{l}\text { Cups/ } \\
\text { day }\end{array}$ & $\begin{array}{c}\text { Temperature } \\
\text { of the tea* }\end{array}$ & $\begin{array}{l}\text { Do you drink } \\
\text { strong tea? } \\
\text { I. Yes 2. No }\end{array}$ \\
\hline \multirow[t]{2}{*}{ 6.2.1 Green tea } & I_ & I_L_ & I_L_ & I_L_ & I_ & $\mathrm{I}$ \\
\hline & I & | I_L & | & |_L & I & I \\
\hline \multirow[t]{2}{*}{ 6.2.2 Oolong tea } & I_ & I_L_ & I_L_ & I_L & I & $\mathrm{I}$ \\
\hline & I I & I_L_ & I_L_ & I_L & I I & I I \\
\hline \multirow[t]{2}{*}{ 6.2.3 Flower tea } & I & I_L_ & | I_L & I_L & I & $\mathrm{I}$ \\
\hline & I_ & I_L_ & I_L_ & I_L & I & I \\
\hline \multirow[t]{2}{*}{ 6.2.4 Black tea } & I I & I_L_ & I_L_ & I_L_ & I I & I I \\
\hline & I I & I_L_ & I_L_ & I_L & I & I_ \\
\hline \multirow[t]{2}{*}{ 6.2.5 Pu'er Tea } & I_ & I_L_ & I_L_ & I_L_ & I_ & I \\
\hline & $\mathrm{I}$ & L_L & L_L & L_L & I & L \\
\hline
\end{tabular}

B

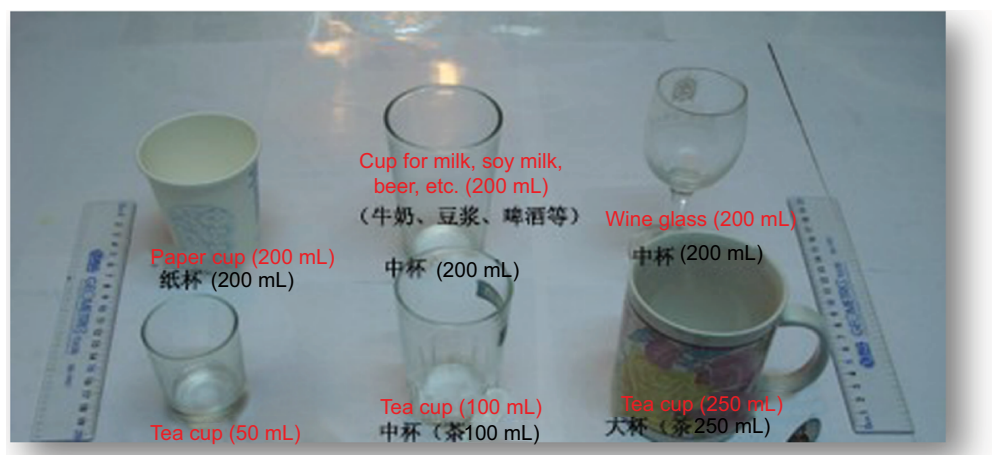

Figure SI (Continued) 


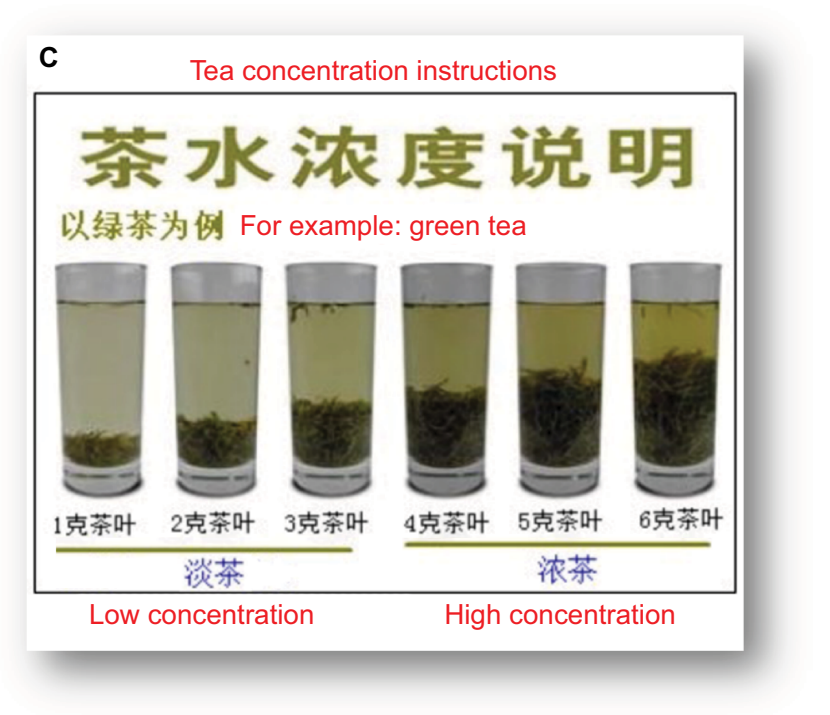

Figure SI Questionnaire of tea drinking and the related sample images.

Notes: (A) The translated questionnaire of tea drinking. *Temperature of the tea: I) very hot - drink immediately (less than I minute); 2 ) hot - wait for a few minutes before drinking ( $1-5$ minutes); and 3) warm - wait for more than 5 minutes before drinking (more than 5 minutes). (B) The related sample images - the display of tea cup size, and $(\mathbf{C})$ the instruction for defining tea concentration (original text with translations included).

Table SI Prevalence of tea drinking habit (with different tea temperature at consumption) by selected risk factors for ESCC in male controls in Taixing, China, 2010-2013

\begin{tabular}{|c|c|c|c|c|c|}
\hline Variables & $\begin{array}{l}\text { Never } \\
\text { N (\%) }\end{array}$ & $\begin{array}{l}\text { Warm } \\
\mathbf{N}(\%)\end{array}$ & $\begin{array}{l}\text { Hot } \\
\text { N (\%) }\end{array}$ & $\begin{array}{l}\text { Very hot } \\
\text { N (\%) }\end{array}$ & $P$-value \\
\hline \multicolumn{6}{|l|}{ Education level } \\
\hline Illiteracy/primary school & $536(72.43)$ & $82(11.08)$ & $86(11.62)$ & $36(4.86)$ & $<0.001$ \\
\hline Junior school and above & $322(52.96)$ & $123(20.23)$ & $124(20.39)$ & $39(6.4 I)$ & \\
\hline \multicolumn{6}{|l|}{ Family wealth score } \\
\hline$\leq$ Median & $428(70.39)$ & $68(11.18)$ & $78(12.83)$ & $34(5.59)$ & $<0.001$ \\
\hline$>$ Median & $430(58.11)$ & $137(|8.5|)$ & $132(17.84)$ & $4 \mid(5.54)$ & \\
\hline \multicolumn{6}{|c|}{ Body mass index 10 years ago } \\
\hline Lean/normal $(<24)$ & $595(65.67)$ & $132(14.57)$ & 134 (14.79) & $45(4.97)$ & 0.023 \\
\hline Overweight/obesity $(\geq 24)$ & $263(59.50)$ & $73(16.52)$ & $76(17.19)$ & $30(6.79)$ & \\
\hline \multicolumn{6}{|c|}{ Sum of missing and filled teeth } \\
\hline None & $207(55.95)$ & $60(16.22)$ & $76(20.54)$ & $27(7.30)$ & $<0.001$ \\
\hline$<6$ & $324(63.65)$ & $89(17.49)$ & $68(13.36)$ & $28(5.50)$ & \\
\hline$\geq 6$ & $322(69.55)$ & $55(11.88)$ & $66(14.25)$ & $20(4.32)$ & \\
\hline \multicolumn{6}{|c|}{ Times of tooth brushing per day } \\
\hline$<2$ & $598(67.04)$ & $116(13.00)$ & $130(14.57)$ & $48(5.38)$ & 0.002 \\
\hline$\geq 2$ & $257(57.11)$ & $88(19.56)$ & $79(17.56)$ & $26(5.78)$ & \\
\hline \multicolumn{6}{|l|}{ Smoking (pack-years) } \\
\hline Never & $239(80.20)$ & $20(6.7 I)$ & $33(11.07)$ & $6(2.01)$ & $<0.001$ \\
\hline$\leq 30$ & $334(64.11)$ & $83(15.93)$ & $75(14.40)$ & $29(5.57)$ & \\
\hline$>30$ & $283(53.80)$ & $101(19.20)$ & $102(19.39)$ & $40(7.60)$ & \\
\hline \multicolumn{6}{|c|}{ Alcohol drinking intensity (g/day) } \\
\hline Never & $423(72.68)$ & $59(10.14)$ & $69(11.86)$ & $31(5.33)$ & $<0.001$ \\
\hline$\leq 80$ & $232(62.37)$ & $69(18.55)$ & $51(13.71)$ & $20(5.38)$ & \\
\hline$>80$ & $202(51.40)$ & 77 (19.59) & $90(22.90)$ & $24(6.11)$ & \\
\hline \multicolumn{6}{|c|}{$\begin{array}{l}\text { Family history of esophageal cancer } \\
\text { among first-degree relatives }\end{array}$} \\
\hline No & $707(64.5 \mathrm{I})$ & $168(15.33)$ & $164(14.96)$ & $57(5.20)$ & 0.078 \\
\hline Yes & $148(59.44)$ & $37(14.86)$ & $46(18.47)$ & $18(7.23)$ & \\
\hline
\end{tabular}

Notes: ${ }^{a}$-values were derived using Kruskal-Wallis test, after excluding the corresponding missing value. 
Subgroup

Cases Controls

Adjusted

P-interaction

N

N

OR $(95 \% \mathrm{Cl})$

Sum of missing and filled teeth

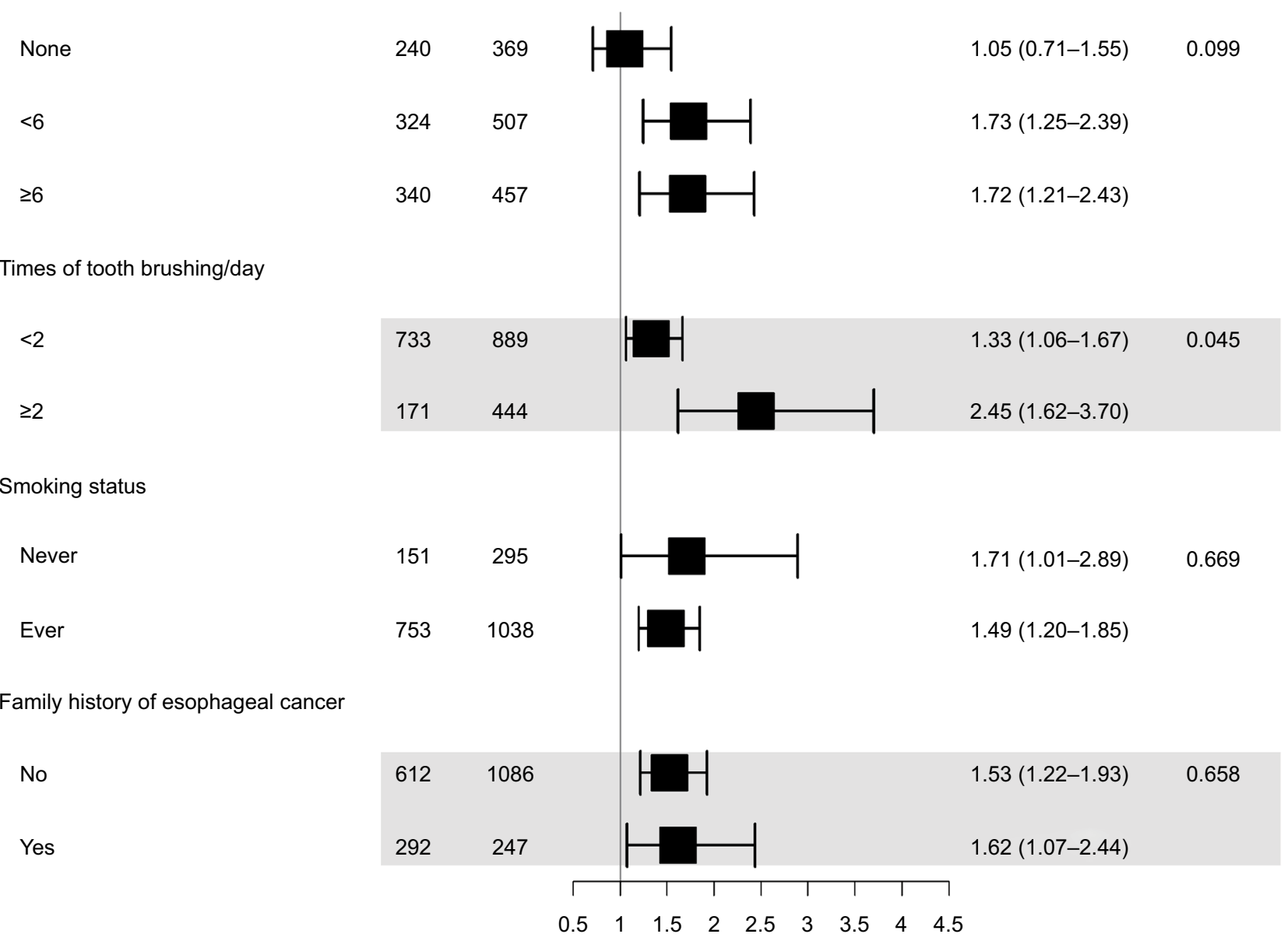

Figure S2 The interaction between green tea drinking and other selected risk factors on the risk of esophageal squamous cell carcinoma by likelihood ratio test.

\section{Publish your work in this journal}

Clinical Epidemiology is an international, peer-reviewed, open access, online journal focusing on disease and drug epidemiology, identification of risk factors and screening procedures to develop optimal preventative initiatives and programs. Specific topics include: diagnosis, prognosis, treatment, screening, prevention, risk factor modification, ogy and biostatistical methods, and evaluation of guidelines, translational medicine, health policies and economic evaluations. The manuscript management system is completely online and includes a very quick and fair peer-review system, which is all easy to use. 